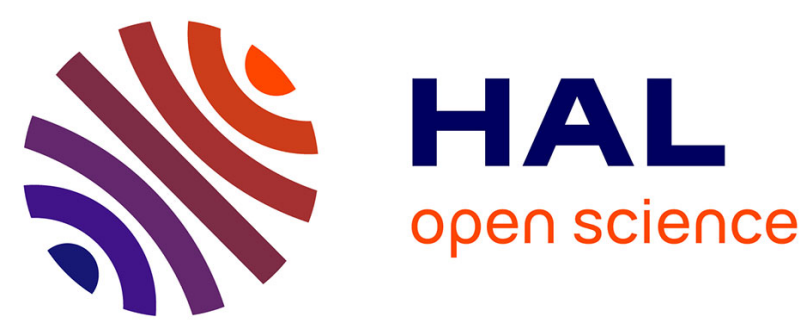

\title{
Understanding Museum visitors' experience through an Eye-tracking study and a Living Lab approach
}

Paola Patricia Parra Morantes, Sonia Andrea Peñarete, Giovanny Arbelaez

Garces, Mauricio Camargo, Laurent Dupont

\section{To cite this version:}

Paola Patricia Parra Morantes, Sonia Andrea Peñarete, Giovanny Arbelaez Garces, Mauricio Camargo, Laurent Dupont. Understanding Museum visitors' experience through an Eye-tracking study and a Living Lab approach. 22nd ICE/IEEE International Technology Management Conference, Jun 2016, Trondheim, Norway. 10.1109/ICE/ITMC39735.2016.9025900 . hal-02083876

\section{HAL Id: hal-02083876 \\ https://hal.univ-lorraine.fr/hal-02083876}

Submitted on 29 Mar 2019

HAL is a multi-disciplinary open access archive for the deposit and dissemination of scientific research documents, whether they are published or not. The documents may come from teaching and research institutions in France or abroad, or from public or private research centers.
L'archive ouverte pluridisciplinaire HAL, est destinée au dépôt et à la diffusion de documents scientifiques de niveau recherche, publiés ou non, émanant des établissements d'enseignement et de recherche français ou étrangers, des laboratoires publics ou privés. 


\section{Understanding Museum visitors' experience through an Eye-tracking study and a Living Lab approach}

\author{
Paola Patricia Parra Morantes, Sonia Andrea Peñarete \\ IntiColombia - ITC Research Group \\ Universidad Nacional de Colombia \\ Bogota, Colombia \\ ppparram@unal.edu.co
}

\author{
Giovanny Arbelaez*, Mauricio Camargo, Laurent Dupont \\ ERPI - EA 3767 \\ Université de Lorraine \\ Nancy, France \\ giovanny.arbelaez-garces@ univ-lorraine.fr
}

\begin{abstract}
User-centered and participatory innovation practices like Living-Labs are nowadays increasingly been used to integrate, design and improve users' experience (UX) in different fields. These methods that had emerged from web and mobile design fields can contribute to the UX in several other fields. Museum visitors experience is a field that can benefit from them. The purpose of this paper is to contribute to the understanding of the museum visitors' experience through the use of tools from UX and usability studies. Among those techniques, field trials studies can help to better understand users' preferences as experience is tested in a real life setting. Exposition curators and managers can then use these understandings to improve visitors' experience. We present a study held at the Museum Aquarium of Nancy-MAN (France). In this study, were evaluated visitors' preferences among four different information supports in an exhibition module using a protocol that combines eyes tracking and an after use survey. Results show that interactive supports, as tablets and screens, even if not preferred by the majority of participants, once engaged with these supports, they interact longer time. Found evidence let us conclude that supports that are different and uncommon are preferred by most of the participants but, they don't keep them engaged for a long time.
\end{abstract}

Keywords- Eye-tracking; User experience; Field trials experiments; Museology; User-centered design;

\section{INTRODUCTION}

User-centered design and participatory innovation practices like Living-Labs promote the user integration through the design cycle [1]-[3]. Their participation allows proposing, exploring and experimenting new ideas and concepts that could be turned into successful innovations. Those approaches have shown to be successful when applied in domains as diverse as health-care, transportation, government and smart-city design among others [2]. The concepts underlying those practices could be useful to the museum community as their tools enable visitors' participation in the development of products, services or exhibitions according to their needs [4]. For instance, the exhibit design process seeks to determine the most effective and engaging ways of content communication. As this process is for the visitors' benefit, why don't engage them in their development? This is what proposals as Museomix try to address [5]. Inspired by Living-Labs and participatory practices, this event-driven community of museum innovators organizes collective events where different professionals and visitors help to co-create the museum experience. Their vision is to create an open-museum that evolves with their users. Providing visitors with "user" driven experiences would allow museums to increase their visits and to develop a stronger relationship with their audience. Museums can increase their relevancy, improve revenues and build new audiences by becoming more user-centric [6], [7].

While these approaches allow to prototype museum visitors' experience through objects and exhibitions, they lacks an understanding of the elements that compose and motivated that experience [8]. Without them it is not possible to know how the user has given sense to that object to build that experience. Our aim is thus to identify and understand how that experience is built under the natural visitor conditions [9]. In the same way that UX can be measured through different behaviors or attitudes (e.g. errors when trying to log into a web page, frustration or delight using an application, visual fixations on a link in a web page), we believe that museum visitors experience can be also measured through visitors' behaviors (i.e. time spend in front of an object, preference of an information support). Measuring those aspects can be useful when trying to improve visitors' experience. That could help museum managers and exhibition designers to make better informed decisions [10], [11].

The museum visit is generally an individual and autonomous activity. Current approaches to understand visitors experience use interviews and questionnaires at the end of the visit to avoid disturbing the natural course of experience [12]. However, this approaches lacks fidelity because the visitors' memory might not be accurate enough to recall the entire visit experience. To overcome those issues Schmitt [8] proposed an interview method where visitors record their visit through a head mounted camera. At the end of the visit an interview is conducted while showing them the video. Recalling the visit through their eyes allows more vivid memories and facilitate the reconstruction of the cognitive process put into action during the visit [8]. We propose to contribute to this approach by using the eye-tracking technology to record the visitors' gaze and analyze directly the elements of interest. For example, to analyze the time spent regarding a certain object or to compare which museum object is preferred. That kind of analysis are the same used in UX and usability studies when the eye-tracking technology is used [10]. 
The reminder of the paper is organized as follows: in Section II, we present the related research on Living labs and UX approaches in museums, UX tools and eye-tracking studies in museums. Section III, describes the proposed research approach, the materials and methods used. Section IV, presents the study findings. Finally, in section V, conclusions and perspectives for future research are presented.

\section{RELATION TO EXISTING WROK}

\section{A. Living-Labs and UX in museums}

The museum community has understood that museums are spaces for knowledge sharing and places for discussion with the community. They are engaging more digital practices to include and better connect with their public as is shown since 1997 in the Museums and the Web annual conference ${ }^{1}$. The community engagement is not only digital but, is also represented by participatory approaches as the Museomix experience [5]. As highlighted by Salgado [4], Museums could foster community participation not only by facilitating the appropriation of information communication technologies (like social media and digital services) but also by engaging the community in the construction of the experience [7]. Living labs offer methods and tools for aiding end users in the construction of new museum experiences. User-centered design and UX methodologies can also contribute to the user participation.

\section{B. UX evaluation tools and Eye-tracking}

The different assessment tools are used for evaluation of user experience. There are two main categories: explicit and implicit methods. The former, concerns methods where the user expresses their thoughts, opinions and emotions. They can be in the form of questionnaires verbal or non verbal. They collect subjective information. The latter, concerns methods that complete what the user can't express directly. In this category the observation of participants or physiological measurements are used. For example, electromyography (EMG), galvanic skin response (GSR), electroencephalography (EEG), heart rate (HR), temperature, respiration rate or eye-tracking [13]. Those methods allow gathering objective data. In our study, given that we want to understand what the user perceives during the visit we decided to use the eye-tracking technology.

There are many eye-tracking technologies, they use a combination of infrared light sources to track where the participant is looking [10]. Those systems can be fixed and attached to a screen or they can be mobile (in the form of glasses). They allow recording participants gaze and superposing the gaze with the visual field of participants. The recording system of eye trackers allows to further analyze users' gaze patterns. This information is very useful and more precise than simply asking users what they were looking because their memory is not very accurate. Application of eyetracking technology ranges from a diversity of fields. For example, cognitive studies, psychology, human-computer interaction, marketing research and medical research [14]-[16].

\footnotetext{
${ }^{1}$ http://www.museumsandtheweb.com/
}

\section{Previous eye-tracking experiments in museums}

There have been some attempts to use the eye-tracking technology in museums to understand visitors' experience. However, those experiences have been more interested in dealing with the problems that the experimentation face when applying the technology. In this section, we discuss some of those works, in order to learn from their experiences and frame our study [17]-[20].

1) The Indianapolis Museum of Art: Gauging the Practical Use of Eye Tracking in Museums

The art museum participated in a research project with the aim to explore whether eye-tracking technology could be useful to museums and to understand how visitors perceived objects in a collection [17]. In this research, over a period of one year, the team used a fixed sensor VT2 EyeTech, they made tree experiments:

- Experiment 1: to track the eye movements of the participants while being guided through a set of tasks, each participant was located sitting in front of a painting and a facilitator told him to perform three tasks 1) to look at the painting without leaving the "frame", 2) to look outside the framework and 3) to look from the outside to the center of the frame.

- Experiment 2: In this test the eye-tracking equipment was calibrated for each participant, which generated greater accuracy in data collection.

- Experiment 3: Used the eye-tracker during a working session with a mediator in which visitors could discuss the artwork and participate with questions.

After performing the data analysis, the study concludes that a fixed device is not able to follow the gaze for occasional visitors walking through space. It has limitations given the distance and the angle of view of the visitor. It is required to calibrate the device for each visitor for future experiments. Even if their approach is not of practical use in galleries, their research suggest that the technology can be used in controlled experiments.

2) The Hatfield Marine Science Center: Eye Tracking on a 3-D Digital Exhibit

This research was conducted with researchers from Oregon State University, in this study the fixed eye-tracker was used to analyze the behavior of two types of visitors (students and teachers) in front of a 3D display [21]. It attempted to assess the levels of attention to different objects projected on the display. The equipment used in the experiment was an SMI RED stand-alone fixed eye-tracker placed on a tripod in front of the display. The experiment concerned 14 visitors, who had no direct contact with the display; given the constraints of the location, the team only accomplished that users observed between $1 / 6$ and $1 / 4$ of the total area of the module. In order to understand how visitors observed the data on the 3D display, two versions of content were proposed: one for the general public and another for specialized audiences with variations in color labels and backgrounds. A total of four analysis variables for each visitor were used. In this process, each user was 
individually calibrated and remained almost motionless in front of the display during the test. At the end of the experience, participants were asked some questions related to the content of the images. The study concludes that, the use of fixed eyetracker makes impossible to fully analyze the interaction in front of this type of display. Natural actions like walking around the display and interaction with buttons or additional information are lost given the restricted field of analysis. The researchers suggested that mobile devices could be more appropriate and allow better data collection.

3) The Deutsches Museum: Exhibiting "The Real Thing”Do Visitors Perceive Authentic Objects Differently?

This research was conducted in collaboration between the museum and the Knowledge Media Research Center ( IWMKMRC ) in Tübingen [18]. The aim was, to analyze how the visitor perceived both a display of a "real object" and a "representation" of it, based on psychological measures. The experiences attempted to understand if the real object had greater credibility, was more fascinating (and therefore get more attention), if it had a stronger argumentative power and if it led to a better understanding of the content of the exhibition. At the time of the experience, the museum had focused on communicating current scientific topics that generate discussion or controversy among the general public. The exhibition concerned the improvement of the body with nanotechnology. There is a psychological argument behind these proposals. For the test, two displays were designed: one with the physical object and another with photographs. In this experiment, a mobile eye-tracker was used. Participants were selected from the usual museum visitors; each participant was calibrated individually, using a reference display with nine points. They were then asked to visit the exhibition naturally. A first part of the experiment was performed showcasing the photographs; the second part presented the real objects. It was observed that the visitor engagement was longer in the object than in photographs of objects. The study concludes that, many variables affect the experiments: the calibration of users, the care of the equipment and the difficulty in performing the analysis of the data (since it must be done manually). There is also a high variance in the fixation times for the visitors; it generates statistical problems in the analysis of the collected data.

The analysis and learning's obtained from those studies allowed us to better prepare or research. The main implication is that to perform an experiment closer to the real visitors' experience a mobile eye tracking has to be used. It is also necessary to better analyze the museum lighting conditions to frame the experiments according to the limitations of the technology. Additionally, the technology seemed to be more appropriate to compare preferences between different information supports.

Our study can be compared to the method proposed by Schmitt [8]. That proposal, even if it doesn't use the eyetracking technology, constitutes a reconstruction of the visitors experience using recordings from their point of view. This principle is very similar to what is attainable with eye-tracking technology. In their methodology, however, is the user who gives meaning to the reconstruction of the visit explaining different points guided by and interview. We propose to use the eye-tracking analysis capabilities to directly analyses different objects in the museum. To test this hypothesis, we propose a study in a museum exhibition where a module composed of four different information supports is analyzed. We analyzed the visitors preferences of these supports and the time spent regarding each one of them.

\section{RESEARCH APPROACH}

The "Museum Aquarium de Nancy" is one of the three most important cultural and scientific establishments in the French city of Nancy. It is managed by the Greater Nancy in cooperation with the Université de Lorraine. Its collection is composed by 57 aquariums, an exhibition of zoology of 570 animals and a reserve of 136700 animals for conservation, research or temporary exhibitions. The museum is divided in two floors. The first floor is dedicated to the marine and aquarium permanent exhibition, the second floor is divided in the permanent zoological exhibition and a space for temporary exhibitions. Between March 2015 and January 2016 a space was dedicated to the temporary exhibition "Ces animaux qu'on mange..." (Those animals that we eat...). In collaboration with the museum manager and staff we decided to evaluate the visitors experience in the temporary exhibition. This exhibition was more interesting to the study compared to the permanent exhibition because the information supports and objects were more diverse. Additionally, the information collected in this space would allow the museum staff to design the new temporary exhibitions by taking into account the study findings.

\section{A. Materials}

In this experiment we worked with two sets of eye-tracker glasses, comprising the following elements:

- A pair of Tobii Pro V1 glasses composed of a video camera that captures the visitor scene and the visitor gaze, IR sensor, an eye motion sensor and a microphone to capture visitor comments.

- A recording unit that, allows recording and storing eyetracker collected data during the test, calibrate the glasses and indicates the battery level.

In addition to this, we used a portable computer to download and analyze the data. We used the "Tobii studio pro" software.

\section{B. Methods}

A pilot exploration was first held. Two visitors equipped with the eye-tracking glasses freely visited the exhibition. The analysis of the recordings allowed us to define the most appropriate spaces and lighting conditions of the room according to the limitations of the device to make the study.

With those parameters a study protocol was defined. We selected four information supports concerning the exhibition module "viande et anatomie" (meat and anatomy). The aim of the experiment was to identify the visitors' preferences among the different information supports proposed in this module of the temporary exhibition on the museum (cf. Fig. 1).

The four information support devices are: 


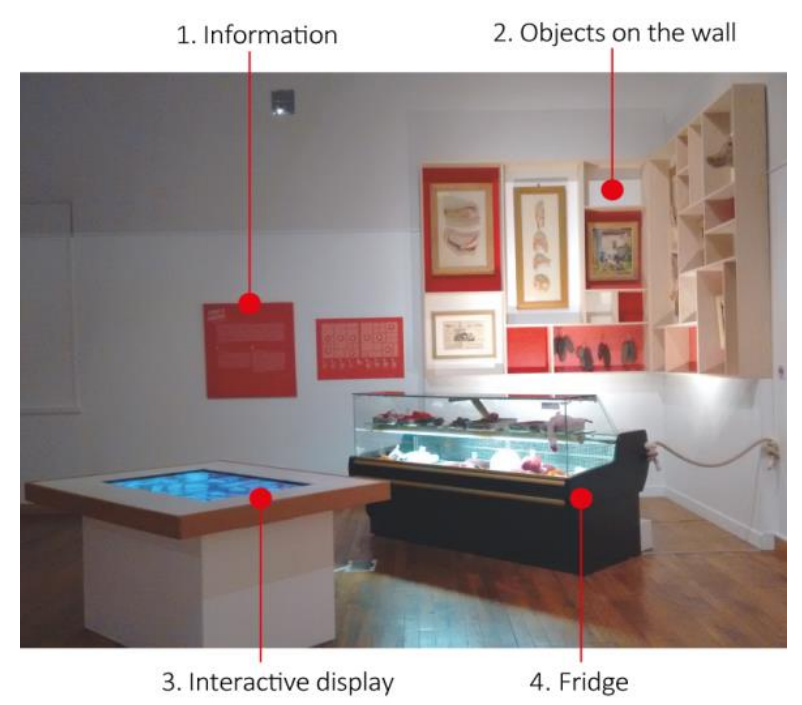

Fig. 1. Museum module and information supports.

1. Panel with descriptive information (text).

2. Objects displayed in a cabinet (wall).

3. Interactive display (tablet).

4. Refrigerator with animal meat in wool (fridge).

The experiment protocol was developed as follows:

- Participants were invited to be part of the study among the museum visitors. Each one signed an authorization form for their image recording.

- Then each participant was equipped with the eyetracker glasses and calibrated individually before starting the tour of the temporary room. The calibration consisted in looking at nine specific points signaled by an IR sensor.

- The visitor was then instructed to freely visit around the temporary exhibition room. No other indication or module was signaled to avoid any bias in the preferences of modules.

- At the end of the visit, the equipment was collected and

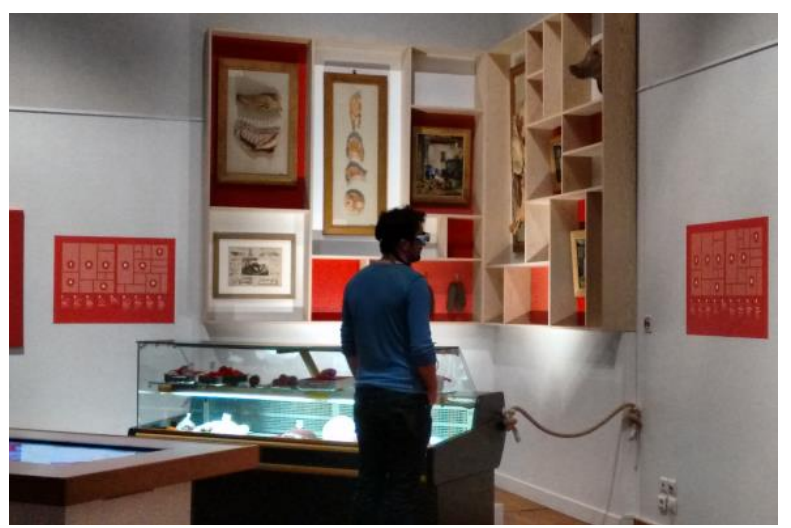

Fig. 2. A participant equipped with eye-tracking glasses during the visit.

\section{Support preference}

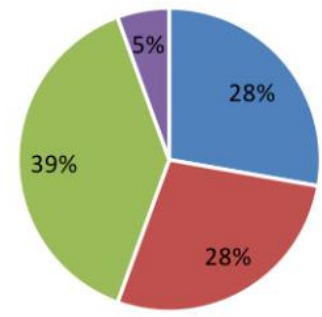

- Tablet $=$ Wall $=$ Fridge $=$ Text

Fig. 3. Visitors' stated support preference.

they were asked to complete a questionnaire about the experience. The questionnaire would allow comparing the data collected with the eye-tracker and the stated preferences of the users.

The test was conducted with a total of 18 adult participants, with an average age of 26 years, 10 of them were men and 8 women. Participants were recruited in two ways: for the first session participants were invited specifically among university students to participate in the test, for the second working session participants were selected randomly from the usual public of the museum. Each session had a duration of 4 hours. Fig. 2 presents a visitor equipped with the eye-tracking glasses during the visit.

\section{FINDINGS}

In the questionnaire visitors were asked to state their preference among the different information supports. The results are presented in Fig. 3. They show that the fridge was preferred by $39 \%$ of visitors, the interactive display (tablet) by $28 \%$, the objects in the wall by $28 \%$ and the informative text by only $5 \%$. When asked for the reasons of those preferences, visitors stated that the fridge is preferred because they feel it was something artistic and interesting. The refrigerator contained representations of animal meat pieces but made in wool as a raw material. The text was perceived as uninteresting because it was only text and seemed too dense. With the other two supports (tablet and wall) the opinions were divided between those how found them uninteresting and not appealing to interact (especially the tablet) and those who found them interesting, but not novel.

Those results, however, are different when we take into account the eye-tracking data. For this analysis we consider that if visitors spend more time looking at a support rather than another is because the former is preferred over the latter. Fig. 4 shows the total times spent looking at the different supports. If we observe the median, the Wall is the preferred support, with the Tablet, Fridge and Text that follows in that order of preference. Those preferences are not concluding because if we observe closer there is a great variability in the Tablet results.

When analyzing in detail the eye-tracking recordings of each participant we found that there is a group ( 8 participants) that engage with the tablet screen and interact with it. This group spends more than 1minute browsing through the content 


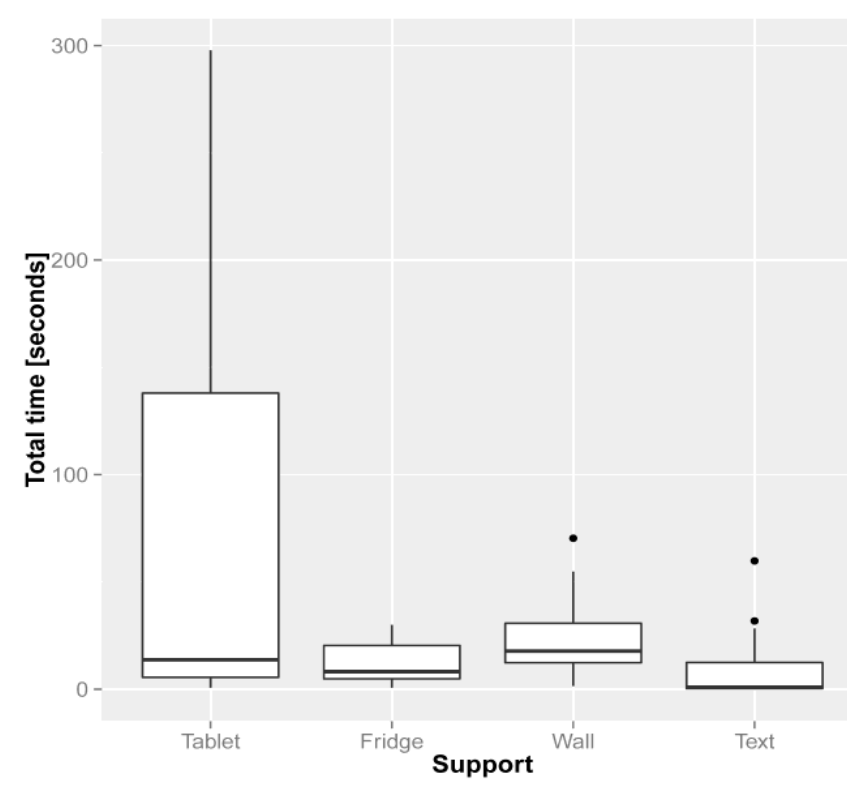

Fig. 4. Visitors' time spend by support.

before changing to another support (Mean=201.6 sec., sd = 91.4). The other participants either ignore the screen or they stop, but don't interact with it (Mean=39.8 sec., $\mathrm{sd}=19.5)$. The users that interacted with the tablet in the questionnaire stated that they found the content attractive and they interacted to know more about the subject.

The results concerning the Wall are consistent both in the questionnaire and the eye-tracking results. The visitors stated that the objects in the Wall were preferred $(28 \%$ second preferred support) and the eye-tracking data show that indeed almost all users spent some time looking that support and if we observe only the median it was the preferred support.

Regarding the Text panels, they don't appear to seem interesting for visitors they don't have a preference for them because they say they are not novel, they are dense and uninviting. Their stated preference corresponds to the eyetracking data.

For the Fridge the results seem contradictory with the visitors' claims as stated in the questionnaire, arguing that it was the preferred support (39\%) compared with time registered by the eye-tracking looking at it. If we only take into account, the median it was the third preferred support. When the eyetracker recording is analyzed it seems that visitors look at the objects placed in the fridge and they seem to be interested, but given that they can only contemplate them they quickly pass to another support and continue with the visit. This compared with the tablet situation seems to indicate that objects that propose an interaction with the visitors might be more interesting.

When analyzing in detail the video recordings, we found that at the end of the exhibition there was another interactive screen that wasn't taken into account in the protocol definition, but users seemed to interact more with this tablet than with the support that was under study. We verified the content and in this screen there was a question that invited visitors to take a quiz to evaluate what kind of animals they eat. The question attracted the attention of the users and they were intrigued to know the answer. For this reason, it would be interesting to further investigate the notions of interactivity in the museum [12].

The aim of this study is to contribute to the understanding of all the factors that affect museum visitors' experience. Having a better understanding of them can help in the design of the exhibition. Evaluating different factors can help identify which information support performs better. In our experimentation, exhibition designers can question the interest of the Text panels, are they really complementary with the Wall objects? An analysis of the eye-tracking recordings for each participant can show whether they consulted the information or they ignored them. In our case, it was mostly ignored. From a manager's perspective that could mean that it is unnecessary and it should be removed to reduce cost, but from the exhibition designer's point of view it is not, it was put there to be complementary to the objects. For the designer the question is rather how to improve the support to make it more relevant for visitors. To help the designer, they can use an approach where different support designs (prototypes) are tested and evaluated with a few visitors helping to choose the one that better conveys the desired experience. The evaluation and the visitors' gathered feedback can be used by the designers to modify their designs. This design approach is uncommon to the museum community, but is commonly used in the usability studies for web design improvement $(\mathrm{A} / \mathrm{B}$ testing for example [22]).

The same approach can be taken with the Tablet screen, different welcome screens can be tested in order to know which one attracts more and keep engaged the visitors.

\section{CONCLUSIONS AND PERSPECTIVES}

We have found that the eye-tracker is a tool that can give us a new perspective on the analysis and understanding of the visitors' experience at the museum since it allows us to observe those elements which attract the attention of visitors. Understanding that the museum audience is diverse and composed by different segments is essential for better understanding of the data. It is suggested that further studies should be focused on specific groups by age, interest, intention of the visit to the museum, among other factors.

We have found that the use of eye-tracking devices should be complemented with the application of questionnaires to better understand the reason and motivations during the visit experience. Using a retrospective think-aloud interview or the method suggested by Schmitt [8] can be complementary to the statistical analysis of the eye-tracking data.

This work is an exploratory study and further analysis is needed. The presented results are partial and exploratory; the statistical analysis is given for information purpose. They show, however, promising results that need further exploration and experimentation. They show the complexity of this type of approaches in experimentation (preparation, participants recruiting, museum agreement...). 
Future work might involve the development of a model containing all the factors that compose the visitors' experience. This might lead light to the understanding of what factors can be modified to construct a better exhibition experience. Further experimentation in other museums is needed.

\section{ACKNOWLEDGMENT}

Authors would like to thanks the MAN managers and collaborators (Pierre-Antoine Gerard, Lucile Guittienne and Arthur Velpry), the ENSGSI Engineering school TEA project students (Givka Aleksov, Mounia El-Boustani, Thomas Leclerc and Marc Wing) and to all the museum visitors that participated in the experiment.

\section{REFERENCES}

[1] S. Kujala, "User involvement: A review of the benefits and challenges," Behav. Inf. Technol., vol. 22, no. 1, pp. 1-16, 2003.

[2] M. Pallot, B. Trousse, B. Senach, and D. Scapin, "Living Lab Research Landscape: From User Centred Design and User Experience towards User Cocreation," in First European Summer School "Living Labs" August, Paris, France, 2010.

[3] L. Dupont, L. Morel, J. Hubert, and C. Guidat, "Study case: Living Lab Mode for urban project design: Emergence of an ad hoc methodology through collaborative innovation," in 2014 International Conference on Engineering, Technology and Innovation (ICE), 2014, pp. 1-9.

[4] M. Salgado, "Museums as Living Labs Challenge, Fad or Opportunity?," The Journal of Community Informatics, vol. 9, no. 3. 05Feb-2013.

[5] "Museomix." [Online]. Available: http://www.museomix.org/. [Accessed: 02-Mar-2016].

[6] J. Leber, "The Future Of Museums Is Reaching Way Beyond Their Walls," co.exist. [Online]. Available: http://www.fastcoexist.com/3044731/the-future-of-museums-isreaching-way-beyond-their-walls. [Accessed: 01-Mar-2016].

[7] M. Salgado, Designing for an open museum: an exploration of content creation and sharing through interactive pieces. Jyväskylä, Finland: Taideteollinen korkeakoulu, 2009.

[8] D. Schmitt, "Apports et perspectives du 'cours d'expérience' des visiteurs dans les musées," ICOFOM Study Ser., vol. 43b, pp. 249-261, 2015.

[9] J. Abascal, M. Arrue, N. Garay, and J. Tomás, "USERfit Tool. A Tool to Facilitate Design for All," in Universal Access Theoretical Perspectives, Practice, and Experience, vol. 2615, N. Carbonell and C. Stephanidis, Eds. Berlin, Heidelberg: Springer, 2003, pp. 141-152.

[10] T. Tullis and B. Albert, Measuring the User Experience. Burlington, MA, USA: Morgan Kaufmann, 2008.

[11] J. Park, S. H. Han, H. K. Kim, S. Oh, and H. Moon, "Modeling user experience: A case study on a mobile device," Int. J. Ind. Ergon., vol. 43, no. 2, pp. 187-196, Mar. 2013.

[12] J. Eidelman, "Visiter les musées: expérience, appropriation, participation," Cult. musées, no. Hors-série, pp. 73-116, 2013.

[13] L. C. da C. B. Silva, A. B. de Oliveira, D. C. Silva, L. C. Paschoarelli, and H. J. C. G. Coury, "Evaluation of reusable cardboard box designs: Biomechanical and perceptual aspects," Int. J. Ind. Ergon., vol. 43, no. 2, pp. 154-160, Mar. 2013.

[14] M. Camargo, R. Bary, N. Skiba, V. Boly, and R. Smith, "Studying the implications and impact of smartphones on self-directed learning under a Living Lab approach,” Int. J. Prod. Dev., vol. 17, no. 1/2, pp. 119-138, 2012.

[15] T. Tien, P. H. Pucher, M. H. Sodergren, K. Sriskandarajah, G.-Z. Yang, and A. Darzi, "Eye tracking for skills assessment and training: a systematic review," J. Surg. Res., 2014.

[16] F. Guo, Y. Ding, W. Liu, C. Liu, and X. Zhang, "Can eye-tracking data be measured to assess product design?: Visual attention mechanism should be considered," Int. J. Ind. Ergon., vol. 53, pp. 229-235, May 2016.
[17] E. Bachta, R. J. Stein, S. Filippini-Fantoni, and T. Leason, "Evaluating the Practical Applications of Eye Tracking in Museums," in Museums and the web, 2012.

[18] K. Eghbal-Azar and T. Widlok, "Potentials and Limitations of Mobile Eye Tracking in Visitor Studies: Evidence From Field Research at Two Museum Exhibitions in Germany," Soc. Sci. Comput. Rev., vol. 31, no. 1, pp. 103-118, Oct. 2012.

[19] E. Mayr, K. Knipfer, and D. Wessel, "In-Sights into Mobile Learning An Exploration of Mobile Eye Tracking Methodology for Learning in Museums," in Researching Mobile Learning: Frameworks, Tools and Research Designs, G. Vavoula, N. Pachler, and A. Kukulska-Hulme, Eds. Bern: Peter Lang, 2009, pp. 189-204.

[20] S. F. Fantoni, K. Jaebker, D. Bauer, and K. Stofer, "Capturing Visitors' Gazes: Three Eye Tracking Studies in Museums," in Museums and the Web, 2013.

[21] S. Rowe, K. Stofer, C. Barthel, and N. Hunter, "Hatfield Marine Science Center: Magic Planet Installation Evaluation Findings," Corvallis, OR, Jul. 2010.

[22] R. Kohavi, R. Longbotham, D. Sommerfield, and R. M. Henne, "Controlled experiments on the web: survey and practical guide," Data Min. Knowl. Discov., vol. 18, no. 1, pp. 140-181, Jul. 2008. 